\title{
Design and Realization of Early Warning System to Monitor Abnormal Parking on Highway
}

\author{
Jiawei Liu ${ }^{1, a}$ and Hongwei Zhang ${ }^{1, b}$ \\ ${ }^{1}$ Southwest University for Nationalities, School of Electrical and Information Engineering, Electrical \\ Engineering \& Automation Chengdu, Sichuan Province 610225 China \\ a201331006041@swun.cn, ${ }^{\text {b7 } 759724337 @ q q . c o m ~}$
}

Keywords: Highway; Monitoring system; Radar positioning; Visibility; Early warning

\begin{abstract}
In order to reduce the malignant vehicle chain rear-end collision accidents under the circumstances of heavy fog and rain and other low-visibility conditions, this paper proposed an early-warning system based on radar positioning to monitor abnormal parking on highway. This system uses radar positioning to monitor the abnormal parking vehicles on highway and builds the network of modules by the use of UDP protocol; then the main control module analyzes and processes the signals collected by the radar and transmits the order to the early-warning light through the network, so as to prompt the rear vehicle to slow down or stop in time by the light signal in the low-visibility conditions and reduce the incidence of accidents. The results proved the effectiveness of the system.
\end{abstract}

\section{高速公路非正常停车监测的预警系统设计}

\author{
刘家玮 ${ }^{1, a}$ ，张红伟 ${ }^{1, b}$ \\ 1. 西南民族大学电气信息工程学院、电气工程及其自动化 中国 四川省 成都市 610225 \\ a201331006041@ swun.cn, ${ }^{\mathrm{b}} 759724337 @$ qq.com
}

摘要: 为了降低在大雾、暴雨等能见度较低的路况下, 高速公路上车辆连环追尾的恶性事故 发生率, 提出一种基于雷达定位的高速公路非正常停车监测的预警系统。本系统利用雷达定 位技术探测高速公路上非正常停驶的车辆, 并采用 UDP 协议进行模块之间的组网, 主控模块 对雷达采集到的信号进行分析处理, 并将命令通过网络传输到预警灯模块, 从而实现在能见 度较低的路况下, 利用光信号提示后方车辆及时减速或停车, 以达到减少事故发生率的目的。 实验结果表明了该系统的有效性。

关键词：高速公路；监测系统；雷达定位；能见度；预警

\section{1. 引言}

随着世界经济的高速发展, 全球高速公路的通车里程不断增加, 但是高速公路的特殊性也带 来了一些新的交通安全问题, 其中由于在大雾、暴雨、夜晚等能见度较低的路况下导致的车 辆连环相撞事故在全球各地都经常发生, 而这种事故必然造成非常严重的生命和财产损失。 在高速公路预警系统方面，美日欧发展较早，中国在最近几年也取得了很大的进步和成果。 借鉴了国内外的高速公路预警系统并研究和设计了新的高速公路预警系统，我们做出了一些 改进和创新, 本系统勿需在车辆上加装任何设备, 并在保证不干扰高速公路正常交通的前提 下，起到能在恶劣天气下有效减少高速公路连环追尾，极大的降低交通事故率。

\section{2. 系统设计}

本系统是基于雷达实时定位、大功率供电、超长线路多节点组网以及计算机软件开发等相关 技术集成构建, 主要由雷达监测系统, 道路数据处理单元, 光纤和无线通信网络以及预警灯 
模块, 输电线路和监控操作中心等5部分组成, 其架构如图1所示。

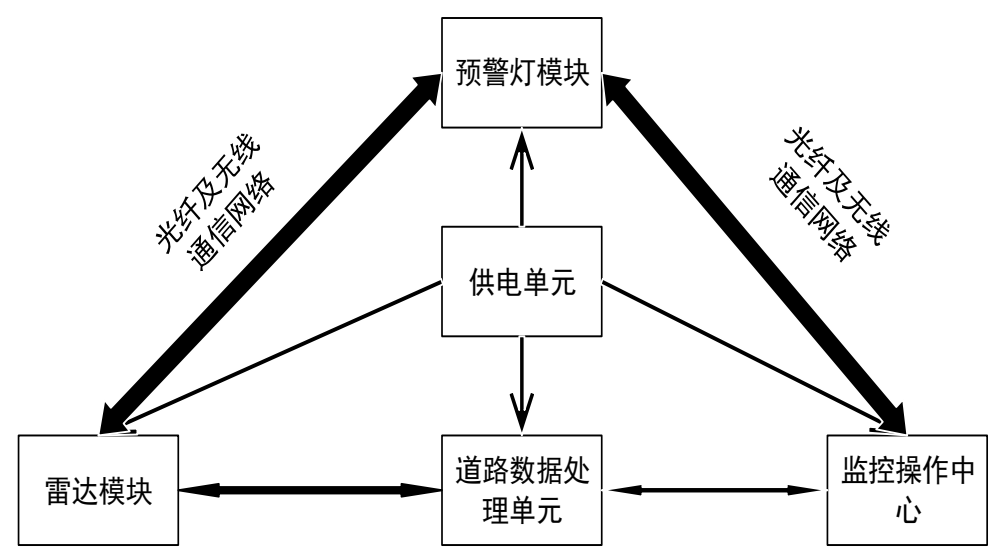

图 1 系统主要架构

在高速公路每隔一定距离安装一个雷达模块, 实时对非正常停车车辆进行监测, 同时每个雷 达模块配套一个道路数据处理单元, 对雷达模块监测所得数据进行快速的分析和计算, 从而 得出目标车辆的坐标位置, 通过光纤网络实时将信息传给下一级的数据处理单元, 直至传到 监控操作中心, 中心可以迅速作出反应, 如果有进一步的处理命令, 通过光纤网络将命令发 送至相应数据处理单元。同时, 以车辆为原点, 数据处理单元将命令通过无线网络传给车辆 后方一定距离内的预警灯, 根据距离远近, 控制预警灯以不同颜色和不同方式进行闪炼。从 而提醒后方车辆, 起到了实时预警的作用。

\section{3. 系统构建关键技术}

3.1. 基于 UDP 的超长线路组网设置

UDP 作为一种广泛使用的轻量级嵌入式 TCP/IP 协议栈, 是专为 8bit 和 16bit 的嵌入式微控 制器设计的微型 TCP/IP 协议栈, 它具有良好的互操作性, 并遵循 RFC 标准。

在使用 UIP 的嵌入式系统的软件体系结构中 UIP 协议栈相当于一个代码库, 它通过一系列的 函数实现与底层硬件和上层应用程序的通信。协议栈和底层硬件和上层应用的关系如图 2 所 示。

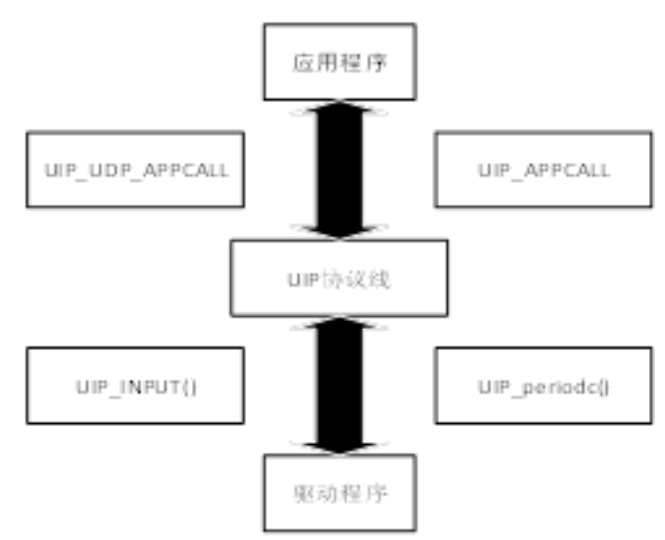

图 2 协议栈关系图

在我们的项目中, 移植了 UIP 到 MCU 中, 采用 UDP 通信方式, 主要用来做一些控制协议的处 理。

3.2. 车辆坐标定位算法

系统当中如何确定车辆的坐标是整个系统需要解决的核心问题之一, 对于雷达所监测到的目 
标位置信息系统无法直接进行识别，需要对信息进行进一步的处理。

首先, 根据雷达传回来的大地坐标的数据, 经过换算换成直角坐标数据。利用 Z 方向的高度 来判断是否车的高度, 如果是一辆车并且在这里停留超过三秒, 那么就会有预警。

先做如下假设:

1. 雷达监测单车道, 并且传回来的数据经过处理之后转换成了雷达数据坐标, 即球坐标数据。

2. 车辆之间的间隔都比较大。

3. 没有超高、超长、超宽的车辆。

系统在高速路上的布置如图 5 所示。

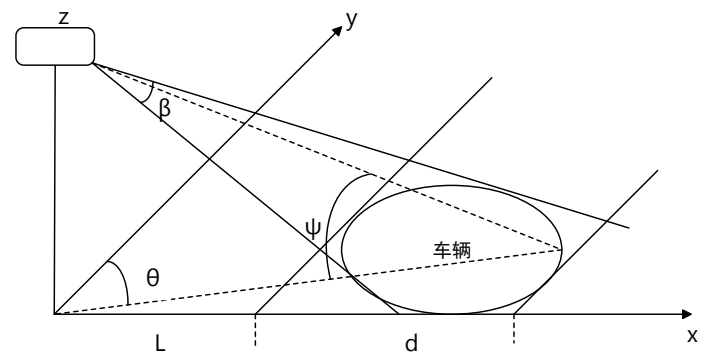

图 3 雷达监测示意图

雷达安装在高度为 $h$ 的标杆顶端。雷达到路面的水平距离为 $\mathrm{L}$, 为雷达径向在道路平面上 $\beta$ 为雷达波束角。 $\Psi$ 为雷达径向与路面的夹角。这里取约等于 90 度。这个假设在本设计中占 有重要的地位。

根据雷达传回来的大地坐标数据, 该数据是基于雷达当前位置所建立的球坐标系的数据。

将球坐标系数据根据下式来转换成直角坐标数据:

$$
\begin{aligned}
& \mathrm{x}=r \sin \psi \cos \theta \\
& y=r \sin \psi \sin \theta \\
& z=r \cos \psi
\end{aligned}
$$

转换为直角坐标之后, 可以根据 $Z$ 轴的高度来判断是否有车, 如果有车并且车的停留时间在 车道上停留超过了三秒，那么将会开启预警系统。整个流程如图 6 所示

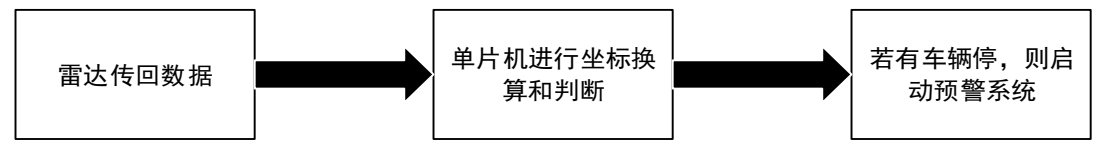

图 4 预警机制流程图

\section{4. 系统验证实例}

为验证系统工作的准确性和稳定性, 对实验样机在实际道路上进行现场测试, 测试时间为 15 天。在实际测试时, 实验路段总长 $300 \mathrm{~m}$, 有三个雷达模块和三个数据处理单元, 同时有 12 个预警灯, 预警灯工作方式如表 1 所示。其中目标距离指目标车辆与预警灯之间的距离, 单 位为 $\mathrm{m}$ 。 
表 1 预警灯工作方式

\begin{tabular}{cc}
\hline 目标距离 $(\mathrm{m})$ & 工作方式 \\
\hline $50--100$ & 红色、每 $1 \mathrm{~s}$ 闪胨一次 \\
$100--200$ & 黄色、每 $1 \mathrm{~s}$ 闪伢一次 \\
$200--300$ & 黄色、每 $2 \mathrm{~s}$ 闪伢一次 \\
\hline
\end{tabular}

在道路测试现场, 记录系统是否正确预警, 以及在系统出现异常故障时及时进行维修, 同时 在实地测试的时候, 发现当车辆较多的时候, 预警的反应时间比车辆稀少的时候的反应时间 要长, 原因是在车辆较多的时候车辆回波之间会影响雷达检测, 为了解决这一问题, 我们在 雷达采集信息之后对信号进行降噪、滤波等处理, 并加入了相应的自适应算法。再次进行实 地测试时，发现上述问题有了良好的改善。最终的测试结果如表 2 所示。

表 2 测试结果

\begin{tabular}{llll}
\hline 时间 (天) & 目标车辆 (辆) & 预警次数（准确率） & 故障次数（故障率） \\
\hline 3 & 23 & $14(100 \%)$ & $0(0 \%)$ \\
6 & 42 & $42(100 \%)$ & $0(0 \%)$ \\
10 & 131 & $130(99.2 \%)$ & $0(0 \%)$ \\
15 & 145 & $144(99.3 \%)$ & $0(0 \%)$ \\
\hline
\end{tabular}

实验测试结果表明, 纠正过的系统的数据传输快速可靠, 预警信息准确, 在测试期间, 故障 率为 $0 \%$, 预警的准确率都超过 $99 \%$, 系统能有效提高高速公路对非正常停车车辆的监测和预 警能力。

\section{5. 结束语}

高速公路非正常停车监测的预警系统的设计及实施方案, 极大降低了高速公路在能见度较低 的路况下的车辆追尾事故率, 这对于我国高速公路信息化、智能化建设具有十分重要的现实 意义。可以预见, 随着信息技术的不断发展和普及, 将会给高速公路非正常停车监测及预警 系统带来更广阔的发展空间。

\section{参考文献}

[1] 代红炎, 龙伟, 李炎炎. 高速公路雨雾监测及车辆安全预警系统研究 $[\mathrm{J}]$. 南京工程学 院学报:自然科学版, 2012, 10(3):55-60.

[2] 李百明. 高速公路汽车追尾预警系统设计 [J]. 电子科技, 2013, 26(2):114-116.

[3] 李建士, 张胜平. 高速公路超速预警系统设计 [J]。交通科技与经济, 2012, 14(3):101-103.

[4] 张媛. 基于 SDH 网络传输系统的高速公路预警系统研究 [D]. 电子科技大学， 2014 .

[5] 石磊. 冰雪条件下高速公路气象检测与预警系统研究 [J]. 公路交通科技: 应用技术版, 2012(9):311-314.

[6] Guan Z G, Liang J, Fan K Q. Research on Intelligent Monitoring and Warning System of Expressway Fog Based on ZigBee [J]. Applied Mechanics \& Materials, 2014, 602-605 (602-605): 1938-1941. 
[7] Yoshihisa Maruyama, Masato Sakaya, Fumio Yamazaki. Effects of earthquake early warning to expressway drivers based on driving simulator experiments [J]. Journal of Earthquake \& Tsunami, 2011, 3(3):261-272.

[8] Wen-Hui L I. The Application of the Internet of Things Technology to Safety Monitoring and Early Warning for Expressway High and Steep Slope [J]. Shanxi Science \& Technology of Communications, 2013.

[9] Zhao X. Monitoring and Early Warning of Dangerous Driving at Expressway Entrance and Exit [J]. Transportation Standardization, 2013.

[10] Wang H D, Gao X X. Method of fuzzy neural network for expressway tunnel health diagnosis and early-warning [J]. China Safety Science Journal, 2014.

\section{References}

[1] Dai Hongyan, Long Wei, Li Yanyan. Study on highway fog monitoring and vehicle safety warning system [J]. Journal of Nanjing Institute of Technology: Natural Science Edition, 2012, 10 (3): 55-60.

[2] Li Bai Ming. The design of the vehicle collision warning system [J]. Electronic Science and technology, 2013, 26 (2): 114-116.

[3] Ling Jianshi, Zhang Shengping. Design of Expressway speeding warning system [J]. Traffic science and technology and economy, 2012, 14(3):101-103.

[4] Zhang Yuan. Research on Expressway early warning system based on SDH network transmission system [D]. University of Electronic Science and technology, 2014.

[5] Shi Lei. Research on highway meteorological detection and early warning system under the condition of ice and snow [J]. Highway traffic science and technology: Application Technology Edition, 2012(9):311-314.

[6] Guan Z G, Liang J, Fan K Q. Research on Intelligent Monitoring and Warning System of Expressway Fog Based on ZigBee [J]. Applied Mechanics \& Materials, 2014, 602-605(602-605):1938-1941.

[7] Yoshihisa Maruyama, Masato Sakaya, Fumio Yamazaki. Effects of earthquake early warning to expressway drivers based on driving simulator experiments [J]. Journal of Earthquake \& Tsunami, 2011, 3(3):261-272.

[8] Wen-Hui L I. The Application of the Internet of Things Technology to Safety Monitoring and Early Warning for Expressway High and Steep Slope [J]. Shanxi Science \& Technology of Communications, 2013.

[9] Zhao X. Monitoring and Early Warning of Dangerous Driving at Expressway Entrance and Exit [J]. Transportation Standardization, 2013.

[10] Wang H D, Gao X X. Method of fuzzy neural network for expressway tunnel health diagnosis and early-warning [J]. China Safety Science Journal, 2014.

作者简介: 刘家玮 (1995一), 男, 河南, 本科, 数字信号处理, 201331006041@swun. cn。 